\title{
ORIENTATION OF DEMAGNETIZED BEES
}

\author{
BY JAMES L. GOULD,* J. L. KIRSCHVINK, $†$ K. S. DEFFEYES, $\dagger$ \\ AND M. L. BRINES $\ddagger$ \\ * Department of Biology, Princeton University, Princeton, N.f. 08544 , U.S.A., \\ $\dagger$ Department of Geological and Geophysical Sciences, Princeton University, \\ Princeton, N.F. 08544, U.S.A., \\ $\ddagger$ Rockefeller University, New York, N.Y. ı0021, U.S.A.
}

(Received 2I September 1979)

\begin{abstract}
SUM MARY
The orientation of honey bee dances is affected by the earth's magnetic field. Honey bees possess localized, well-oriented, stable and superparamagnetic domains of magnetite. Four lines of evidence suggest that the superparamagnetic domains of bees are more likely to be involved in magnetic field detectors than the stable domains. (I) Although the stable domains vary widely in size and number between bees, approximately $2 \times 10^{8}$ superparamagnetic domains are found reliably in all bees, and are restricted to the relatively narrow size range of $300-350 \AA$. This suggests that the superparamagnetic domains are more likely to have a biological function. (2) Behavioural observations of dances in null fields are difficult to reconcile with a stable-domain detector but are clearly predicted by many superparamagnetic detector models. (3) When honey bees are demagnetized, their ability to orient to the earth's field is unaffected. This suggests that the detector either utilizes the superparamagnetic domains or depends on aligned anisotropic stable domains processed without regard to magnetic polarity. (4) Bees that have only superparamagnetic domains are able nevertheless to orient to the earth's magnetic field, a phenomenon which indicates that permanent domains may not be required for detection.
\end{abstract}

\section{INTRODUCTION}

Although the orientation of a variety of animals is, at the very least, affected by the earth's magnetic field (e.g. Keeton, 1972; Wiltschko \& Wiltschko, 1972; Walcott \& Green, 1974; Blakemore, 1975; Bookman, 1977; Phillips, 1977; Emlen et al. 1976; Walcott, 1978 ), almost nothing is known about how terrestrial animals detect it. There are at least three general mechanisms which might be used (Gould, Kirschvink \& Deffeyes, 1978). The first requires that animals possess a device to measure the charge separation that is induced when it is moved through the earth's field. This is probably the system employed by elasmobranch fish (Kalmijn, 1978), where the salt water environment provides a favourable return path for induced current flow. A second method of detection might be accomplished by using permanent magnets that, 
like compass needles, produce a measurable torque as they attempt to twist into alignment with the earth's field. This is the mechanism used by magnetotactic mud bacteria (Kalmijn \& Blakemore, I978). The third strategy would require that animals have paramagnetic substances - molecules which, in the presence of an external field, produce additional magnetism parallel to the external field. An important and generally unappreciated variant of this hypothesis proposes that animals possess superparamagnetic domains - tiny, ferromagnetic crystals which easily align their magnetism in the direction of the applied field at room temperature. Evidence presented here favours this last possibility.

Honey bees are sensitive to the earth's magnetic field. For example, normal dances on vertical comb show small $\left(\leqslant 20^{\circ}\right)$ errors in direction (Missweisung), which depend on the orientation of the earth's field (Lindauer \& Martin, I 968 ). When a hive is left on its side for at least 2 weeks, the resulting horizontal dances (in the absence of other cues) become oriented to the eight points of the magnetic compass (Martin \& Lindauer, I977; Brines, I978). Both of these effects disappear when the earth's field is cancelled.

We reported previously that honey bees possess tranversely aligned stable and superparamagnetic domains of magnetite (Gould et al. 1978). In the process of characterizing these magnetic crystals, we measured the external field strength required to realign all of the domains. We found that the number of domains realigned increased steadily with increasing applied field strength until complete saturation was reached at $1500 \mathrm{Oe}$-approximately 3000 times the strength of the earth's field. This measurement provides the information necessary to test most variants of the permanent magnet hypothesis. If the normally transversely aligned domains were to have their orientations randomized, a permanent-magnet detector which depends on that relative alignment should be deactivated. Saturation and randomization should have no effect on either an inductive or (super)paramagnetic system. In the course of attempting to demagnetize our bees, three other lines of evidence reflecting on the nature of the detector came to light.

\section{METHODS}

In order to demagnetize a bee it is necessary to put it into an alternating field (AF) of at least $\mathrm{I} 500 \mathrm{Oe}$ so as to force the magnetism of all the domains to track the applied field, and then to decrease the field strength slowly so that first the stronger, and then ever weaker, domains stop being driven one by one, and come to rest in a stable orientation determined by the polarity of the last external field strong enough to reverse them. Hence, their directions become randomly dispersed in space and no net magnetism results.

We used a 2100 Oe coil tuned to $60 \mathrm{~Hz}$ to demagnetize our bees. The coil was fabricated by the coil shop of the Princeton University Plasma Physics Laboratory. It consisted of $295^{6}$ turns of no. I 2 wire on an ${ }_{1} \cdot 5 \mathrm{~cm}$ air core, with water cooling between the inner and outer 18 layers. The resistance of the coil was $10.62 \Omega$ while its inductance was $\mathrm{I} \cdot \mathrm{I} 68 \mathrm{H}$. We tuned the coil to near $60 \mathrm{~Hz}$ by adding $5.8 \mu \mathrm{F}$ of capacitance in series. We ran the coil at $10.5-110 \mathrm{~A}$ from a Variac. The coil was mounted inside a ten-coil Helmholtz system (McElhinny, Luck \& Edwards, I97I) to 
cancel the earth's field and thereby prevent any d.c. bias on the domains as they came to rest. Bees on their way out of the hive would walk into the coil, spiral through $360^{\circ}$, and then walk out of and away from the coil and into a large null field (10 ${ }^{-4} \mathrm{Oe}$ over a diameter of $15 \mathrm{~cm}$ and $10^{-3}$ Oe to $50 \mathrm{~cm}$ ). The wide, even distribution of domain sizes inferred from the smooth Curie temperature, saturation magnetization, and low temperature warming remanence curves (Gould et al. 1978) indicated no abrupt changes in the distribution of domain strengths, and even using the steepest part of the steepest curve, no more than $0.2 \%$ of the domains would ever drop out in any single field cycle. As a result, the demagnetizing process was free of any discontinuities which might have tended to bias the net domain orientation. We confirmed that the demagnetization worked by measuring six of the treated bees in a cryogenic magnetometer. The mean remanence was $0.01 \times 10^{-6}$ e.m.u. (against a background of $0.0 \mathrm{I} \times \mathrm{IO}^{-6} \mathrm{e}$.m.u.) compared to a remanence of $0.34 \times 1 \mathrm{O}^{-6} \mathrm{e} . \mathrm{m} . \mathrm{u}$. measured in untreated bees from the same colony.

The assay for magnetic field sensitivity we used was the orientation of horizontal dances in a non-magnetic hive which had been on its side for 4 weeks. We trained individually numbered foragers to a station $240 \mathrm{~m}$ away, and videotaped their dances under red light (to which bees are blind). Bees which showed good orientation to the earth's field were then selectively demagnetized as they left the hive. Their dances were taped on each subsequent return from the feeder.

\section{RESULTS AND DISCUSSION}

\section{(1) Demagnetization}

Demagnetization had no effect on the ability of bees to orient to the earth's magnetic field in this context (Fig. I). We conclude therefore either that the natural remanence in bees is not used in a permanent magnet detector for their horizontal dance orientation or that the permanent domains are used in a way which does not take advantage of the consistent relative alignment of their magnetic moments. The most plausible models for such a single-domain system would require domains which, like those in pigeons (Walcott, Gould \& Kirschvink, 1979), are anisotropic (i.e. elongate in the simplest case), and whose axes of anisotropy are aligned. Upon demagnetization, such domains will take up new moments either in the former direction, or directly opposite to it. If the detector itself is designed only to measure the amount of torque and to ignore its direction, this random, $180^{\circ}$ flipping of half of the domain moments, which would result from demagnetization, might have no effect on the output (J. I. Kirschvink \& J. L. Gould, in preparation). Of course, such a detector strategy would confuse north and south, but then so do bees (Fig. I) and, for that matter, birds (Walcott \& Green, 1974). In comparing this 'axial' variant of the single-domain detector strategy with the superparamagnetic alternatives, we are inclined to favour the superparamagnetic hypothesis on the basis of the three observations which follow. 


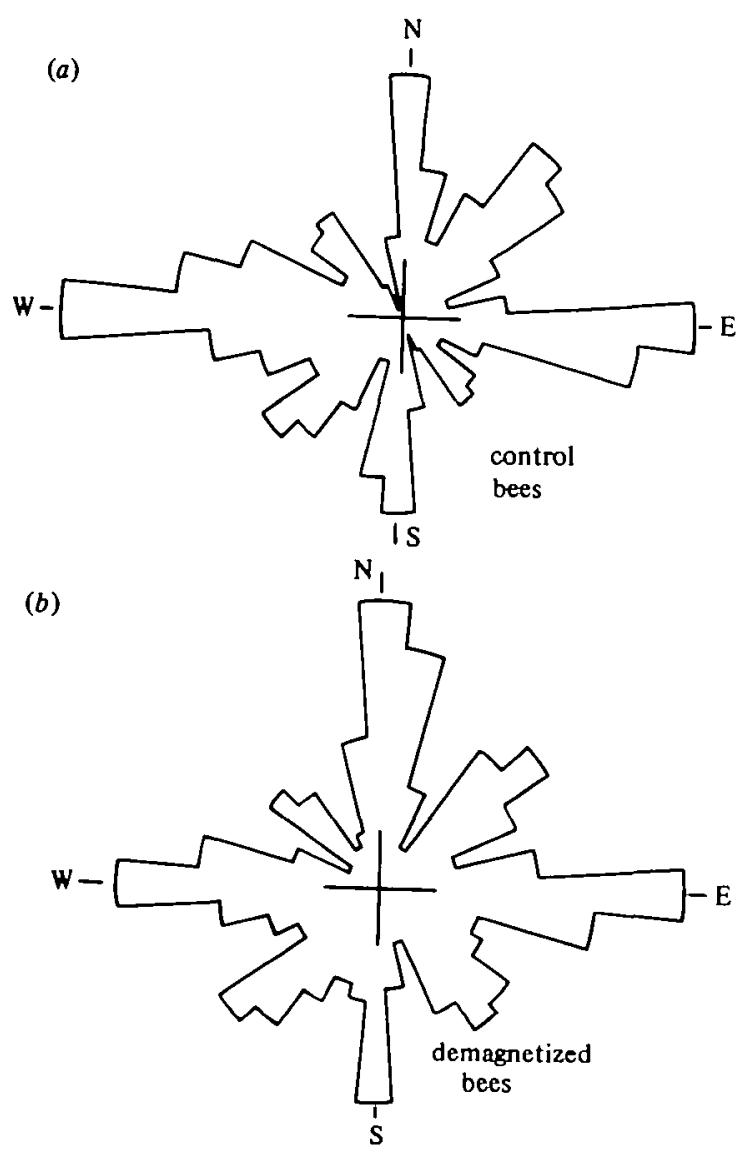

Fig. I. Directions of individual dance cycles for 12 bees under red light on a horizontal surface in an undisturbed earth's magnetic field. Magnetic directions are indicated. Each arm of the crosses in the center of the figures represents five dances. ( $a$ ) Before treatment, the dances are oriented to the eight points of the magnetic compass $(n=438)$. (b) After demagnetization with a $2100 \mathrm{Oe}$. AF coil in a null field, dance orientations continue to be oriented to the eight compass points $(n=395)$.

\section{(2) Bees without remanence}

Some bees, including two of the control bees which orient their dances accurately to the earth's magnetic field in this experiment but were not demagnetized before being measured, possess no detectable permanent domains (i.e. both were measured at the background level of $0.01 \times 1^{-6}$ e.m.u.), while we found a substantial superparamagnetic remanence in all adult bees examined (Gould et al. 1978). This suggests that the stable domains are unnecessary for magnetic field orientation.

\section{(3) Superparamagnetic remanence}

The superparamagnetic remanence was always at least as large as the permanent one, and was due largely or exclusively to magnetite. We observed these superparamagnetic domains by cooling the bees in liquid nitrogen to make the crystals magnetically stable. We then induced a remanence with a $3000 \mathrm{Gs}$ magnet and watched 


\section{Orientation of demagnetized bees}

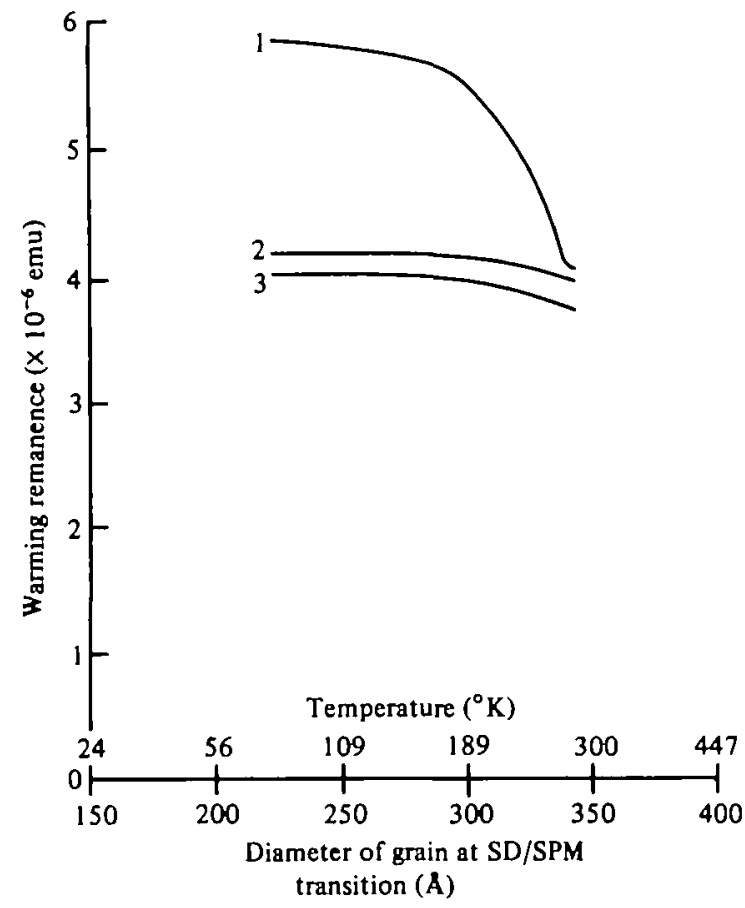

Fig. 2. Superparamagnetic grains in a worker bee. The relaxation time for a magnetic domain increases exponentially as the temperature is lowered. Thus, some grains which are superparamagnetic at room temperature $\left(\sim 300^{\circ} \mathrm{K}\right)$ will behave as single domains capable of holding remanence at liquid-nitrogen temperature $\left(-77^{\circ} \mathrm{K}\right)$. Conversely, any net remanence held by these domains at low temperature will disappear as they warm in field-free space across their superparamagnetic/stable domain transition (their blocking temperature). The temperature at which the remanence is lost indicates the grain size responsible. To check for superparamagnetic grains the bees were cooled to $77^{\circ} \mathrm{K}$ in the presence of a strong magnetic field ( $\sim 3000 \mathrm{Gs}$ ), forcing all the domain moments into alignment. Curve 1 shows the remanence of one bee as it warmed up inside the field-free region of a cryogenic magnetometer. Most of the remanence is lost as the stable/superparamagnetic size transition boundary goes from 300 to $350 A$. Since a magnetite sphere $325 A$ in diameter has a moment of about $8.6 \times 10^{-15}$ e.m.u., about $2 \times 10^{\prime}$ of them are necessary to account for the lost remanence. Curves 2 and 3 show subsequent warming cycles without exposure to the strong magnet.

the remanence decay as the bee warmed up in a cryogenic magnetometer. Our calculations based on this curve (J. L. Kirschvink \& J. L. Gould, in review) indicate that the superparamagnetic domains are confined to the narrow size range of $300-$ $35^{\circ} \AA$, and number about $2 \times 10^{8}$ per bee (Fig. 2 ). In contrast to the widely variable number and size of stable domains, we believe the measurements of superparamagnetic remanence suggest a biological function.

\section{(4) Dances in null fields}

The Missweisung effect of the earth's field on vertical dances cannot be accounted for easily with transversely aligned permanent domains. This is because Missweisung disappears when bees dance along field lines (Lindauer \& Martin, 1969). In these dances, the fields of the domains would be orthogonal to the earth's field, the 
orientation which would cause maximum torque on permanent magnets. Yet a nulf field, which must result in no torque, also causes Missweisung to disappear. It is difficult to understand how both a maximum and minimum detector response could produce the same behavioural output. More puzzling still, bees dancing perpendicular to the earth's field lines would have little or no torque on their magnets - a situation similar to the null field circumstance - and yet display maximum Missweisung (Lindauer \& Martin, 1969). Of course, it is remotely conceivable that bees are programmed to sense field direction only while turned $90^{\circ}$ to the eventual dance angle. Our behavioural observations, however, indicate that bees often take up appropriate dance directions without turning. In fact, bees which have begun a dance cycle orient to a light which is then switched off in the middle of their waggle run will often reorient the remaining part of the run to the nearest compass point. Since this realignment is always less than $25^{\circ}$, occurs in mid-run, and results as often in the bees' dancing along the earth's magnetic field lines as orthogonal to them, it suggests that dancing bees are taking in and using magnetic information continuously, just as they do when orienting the dance to visual or gravitational cues. No plausible stable domain system can account for this.

Superparamagnetic domains, on the other hand, could be arranged to mimic the Missweisung behaviour in many ways (J. L. Kirschvink \& J. L. Gould, in review). Suppose, for example, the crystals are anisotropic with their long axes transversely oriented, as seems to be the case with the permanent domains. Assume further that they are spaced close enough to interact with each other and are arranged around, say, a pressure sensor. With the field transverse to the bee (the maximum Missweisung condition), the long axis of the crystals would be aligned with the field, and a maximum paramagnetic field would be induced. In this instance the superparamagnetic crystals would repel each other, creating an effect on the pressure sensor which would disappear in a null field. This is consistent with the behavioural data. When such a bee faces along the field lines such that the axis of its superparamagnetic crystals would be perpendicular to the earth's field, a minimum paramagnetic field would be induced, and cancelling the external field would have little or no effect. Again, this mimics the observed behaviour. Several other superparamagnetic models are possible, including ones which do not assume any shape anisotropy (J. L. Kirschvink \& J. L. Gould, in review), and many yield sensitivities of $10^{-4} \mathrm{Oe}$ or better, a value which agrees with the most extreme sensitivity claimed for animals.

Although these data indicate that bees are not using their permanent magnets in their dance orientation, they say nothing about the sensory mechanisms which must underlie their reported ability to set their circadian rhythms to small regular daily variations in the earth's magnetic field (Martin \& Lindauer, 1977). Such an ability could be accounted for plausibly by a permanent-domain detector, and it is conceivable that bees have two systems for extracting magnetic field information. This possibility can be tested by demagnetizing the magnetically entrained bees.

Again, though these data tend to exclude a stable-domain mechanism for dance orientation, they do not thereby necessarily prove that the superparamagnetic domains are being used. It is conceivable that these localized, well-aligned domains of magnetite have no function at all; but the recent discovery of a small, innervated, 
magnetite-rich tissue in homing pigeons (Walcott et al. 1979) lends support to our hypothesis that these concentrations of magnetic materials are not accidental, but rather play a role in orientation.

Although it is very unlikely, the induction hypothesis cannot yet be ruled out in bees. Now that the permanent magnet possibility has been virtually excluded, however, a relatively conclusive test of the paramagnetic theory is possible. Small magnets glued to the bodies of foragers should disrupt their horizontal dances if the magnet is close enough to a paramagnetic detector and properly aligned. The static field of such a fixed magnet should not affect an induction detector in the body. (A leg or antennal detector would be affected, but the range of effective magnet locations would separate the two possibilities.) Distinguishing between an ordinary paramagnetic system and a detector using superparamagnetic domains of magnetite would be less easy. Finding the detector just where we also find the magnetite, though, would be a very suggestive first step, and would follow from the glued-magnet experiment. In the end, however, only detailed anatomical and physiological investigations can provide conclusive evidence of how the magnetic field detector in bees works.

We thank C. Bushnell of the PPL coil shop for designing the coil, L. Martin of the MBL electronic shop for helping in the final stages of tuning the coil, C. Denham and A. Chave of Woods Hole Oceanographic for helping us with the cryogenic magnetometer measurements, R. Dahl, E. Tyner, C. G. Gould and K. Schenck for help in setting up and running the experiments, C. G. Gould for help with the manuscript, and W. G. Quinn for prodding us to do the experiments in the first place. This research was supported by NSF grant BSN 76-or653 to J. L.G.

\section{REFERENCES}

BOOKMAN, M. A. (1977). Sensitivity of homing pigeons to an earth-strength magnetic field, Nature, Lond. $267,340-342$.

BLAKEMORE, R. (1975). Magnetotactic bacteria, Science, N.Y. 190, 377-379.

BRINES, M. L. (I978). Skylight polarization patterns as cues for honey bee orientation. Thesis, Rockefeller University, New York.

Emlen, S. T., Wiltschko, W., Demong, N., Wiltschko, R. \& Bergman, S. (1976). Magnetic direction finding in migratory indigo buntings. Science, N.Y. x93, 505-508.

Gould, J. L., Kirschvink, J. L. \& DefFeyes, K. S. (1978). Bees have magnetic remanence. Science, N.Y. 201, 1026-1028.

Kalmijn, A. J. (1978). Experimental evidence of geomagnetic orientation in elasmobranch fishes. In Animal Migration, Navigation, and Homing (ed. K. Schmidt-Koenig and W. T. Keeton), pp. 348354. New York: Springer-Verlag.

Kalaijn, A. J. \& Blakemore, R. (1978). Magnetic hehaviour of mud bacteria. In Animal Migration, Navigation, and Homing (ed. K. Schmidt-Koenig and W. T. Keeton), pp. 354-355. New York: Springer-Verlag.

KeEton, W. T. (1972). Effects of magnets on pigcon homing. In Animal Orientation and Navigation (ed. S. R. Galler et al.) pp. 579-594. Washington, D.C.: NASA.

Kirschvink, J. L. \& GovLd, J. L. Organic magnetite: a ferrimagnetic basis for magnetic field orientation in anımals. F. comp. Physiol. (in preparation).

Lindauer, M. \& Martin, H. (1968). Die Schwereorientierung der Bienen unter dem Einfluss der Erdmagnetfeldes. $Z$. vergl. Physiol. 60, 21 9-243.

Martin, H. \& Lindauer, M. (1977). Der Einfluss der Erdmagnetfelds und die Schwereorientierung der Honigbiene. f. comp. Plyysiol. 122, I45-187.

McElhinny, M. W., Luck, G. R. \& Edwards, D. (1971). A large volume magnetic field fiee space. Pure appl. Geophys. 90, 126-1 30. 
Phillips, J. (1977). Use of the earth's magnetic field by orienting cave salamanders. $\mathcal{J}$. comp. Physio/ I2I, 273-288.

WaLCOTT, C. (1978). Anomalies in the earth's magnetic field increase the scatter of pigeons' vanishing bearings. In Animal Migration, Navigation, and Homing (ed. K. Schmidt-Koenig and W. T. Keeton), pp. I43-I 51 . New York: Springer-Verlag.

Walcott, C., Gould, J. L. \& Kirschvink, J. L. (1979). Pigeons have magnets. Science, N. Y. 205, 1027-1029.

IVALCOTT, C. \& GrefN, R. P. (I 974). Orientation of homing pigeons altered by a change in the direction of an applied magnetic field. Science, N.Y. 184, 180-182.

Wiltschko, W. \& Wiltschko, R. (1972). Magnetic compass of European robins. Science, N.Y. 176, 62-64. 